G. QIU

KODAI MATH. J.

16 (1993), 318-326

\title{
ON A CONJECTURE OF C.C. YANG FOR THE CLASS $F$ OF MEROMORPHIC FUNCTIONS
}

\author{
BY QIU GANGDI
}

\begin{abstract}
In this paper, we give a positive answer to a conjecture of C.C. Yang for the class $F$ of meromorphic functions, and improve a result of C.C. Yang.
\end{abstract}

Key words: Meromorphic function, Deficient value, Unicity.

\section{Introduction and Main Results.}

In this paper, we use the signs as given in Nevanlinna theory [3], let $E$ denote a set of positive real number with a finite linear measure, which is not necessarily the same at each time it occurs. If the two meromorphic functions $f$ and $g$ have the same $a$-points and multiplicities, we denote it by

$$
E(a, f)=E(a, g) .
$$

In 1977, C. C. Yang proved the following theorem:

THEOREM A ([1]). Let $F$ denote a class of meromorphic functions with the form as $f=\mu_{1} e^{\alpha}+\mu_{2}$, where $\alpha$ is a nonconstant entire function with finite order, $\mu_{1}(\not \equiv)$ and $\mu_{2}$ (非onst.) are two meromorphic functions with finite order, satisfying

$$
T\left(r, \mu_{i}\right)=o\left\{T\left(r, e^{\alpha}\right)\right\}, \quad(i=1,2) .
$$

Suppose $c_{1}, c_{2}$ are two distinct finite complex numbers, $f \in F$ and $g \in F$. If

then $f \equiv g$ or

$$
E\left(c_{\imath}, f\right)=E\left(c_{\imath}, g\right), \quad(i=1,2)
$$

$$
\begin{aligned}
& f=\frac{c_{2}-c_{1} \lambda(z)}{1-\lambda(z)}-\frac{\left(c_{1}-c_{2}\right)^{2} \lambda(z)}{1-\lambda(z)} \cdot \frac{1}{h(z) \cdot e^{\phi(z)}}, \\
& g=\frac{c_{1}-c_{2} \lambda(z)}{1-\lambda(z)}+\frac{h(z) e^{\phi(z)}}{1-\lambda(z)}
\end{aligned}
$$

Received July 6, 1992; revised January 6, 1993. 
where $\phi(z)$ is a nonconstant entire function, $\lambda(z)$ (非onst.) and $h(z)$ are two meromorphic functions, satısfying

$$
T(r, \lambda)=o\left\{T\left(r, e^{\phi}\right)\right\}, \quad T(r, h)=o\left\{T\left(r, e^{\phi}\right)\right\} .
$$

Further, he conjectured that theorem A also holds for the class of meromorphic functions with the form as

$$
f=\mu_{1} e^{\alpha}+\mu_{2},
$$

where $\alpha$ is a nonconstant entire function, $\mu_{1}(\not \equiv 0)$ and $\mu_{2}(\not \equiv$ const.) are two meromorphic functions, satisfying

$$
T\left(r, \mu_{\imath}\right)=o\left\{T\left(r, e^{\alpha}\right)\right\} . \quad(\imath=1,2)
$$

In the present paper, we give a positive answer to C. C. Yang's conjecture. More generally, the following results are obtained.

THEOREM 1. Let $f, g, \mu$ and $\lambda$ be nonconstant meromorphic functions, satıs. fying

$$
T(r, \mu)=o\{T(r, f)\}, \quad T(r, \lambda)=o\{T(r, g)\} .
$$

If $E(\infty, f)=E(\infty, g), E(\mu, f)=E(\lambda, g)$, and

$$
\delta(0, f)+\Theta(\infty, f)>\frac{3}{2}, \quad \delta(0, g)+\Theta(\infty, g)>\frac{3}{2},
$$

then

$$
\frac{f}{\mu}=\frac{g}{\lambda} \quad \text { or } \quad f \cdot g=\mu \cdot \lambda
$$

THEOREM 2. Let $f, g, \varphi_{1}, \varphi_{2}, h_{1}$ and $h_{2}$ be nonconstant meromorphic functrons, satisfying

$$
T\left(r, \varphi_{i}\right)=o\{T(r, f)\}, \quad T\left(r, h_{\imath}\right)=o\{T(r, g)\}, \quad(i=1,2) .
$$

If $E(\infty, f)=E(\infty, g), E\left(\varphi_{\imath}, f\right)=E\left(h_{\imath}, g\right),(i=1,2)$ and

$$
\delta(0, f)+\Theta(\infty, f)>\frac{3}{2}, \quad \delta(0, g)+\Theta(\infty, g)>\frac{3}{2},
$$

then

$$
\frac{f}{\varphi_{1}}=\frac{g}{h_{1}} \quad \text { and } \quad \frac{\varphi_{1}}{h_{1}}=\frac{\varphi_{2}}{h_{2}}
$$

or

$$
f \cdot g=\varphi_{1} \cdot h_{1} \quad \text { and } \quad \varphi_{1} \cdot h_{1}=\varphi_{2} \cdot h_{2} .
$$

COROLlary. The conjecture of C.C. Yang is ture. 


\section{Some Lemmas.}

LEMMA 1 ([2]). Let $f_{\mathcal{J}}(j=1,2, \cdots, n)$ be $n$ linearly independent meromorphic functions with $\sum_{j=1}^{n} f_{j} \equiv 1$, then

$$
\begin{aligned}
T\left(r, f_{j}\right)< & \sum_{\imath=1}^{n} N\left(r, \frac{1}{f_{i}}\right)+N\left(r, f_{j}\right)+N(r, D) \\
& -\sum_{i=1}^{n} N\left(r, f_{\imath}\right)+o\{T(r)\}, \quad(r \notin E ; j=1,2, \cdots, n)
\end{aligned}
$$

where $T(r)=\max _{1 \leq j \leq n}\left\{T\left(r, f_{j}\right)\right\}$,

$$
D=\left|\begin{array}{cccc}
f_{1} & f_{2} & \cdots & f_{n} \\
f_{1}^{\prime} & f_{2}^{\prime} & \ldots & f_{n}^{\prime} \\
\ldots \ldots \ldots \ldots \ldots \ldots \ldots \ldots \ldots \\
f_{1}^{(n-1)} & f_{2}^{(n-1)} & \ldots & f_{n}^{(n-1)}
\end{array}\right| .
$$

LEMMA 2 ([4]). Let $f_{1}$ and $f_{2}$ be two nonconstant meromorphic functions, and let $\alpha_{1}(\not \equiv 0)$ and $\alpha_{2}(\not \equiv 0)$ be two meromorphic functions, it satisfies

$$
T\left(r, \alpha_{\imath}\right)=o\{T(r)\}, \quad(r \notin E ; i=1,2)
$$

where $T(r)=\max \left\{T\left(r, f_{1}\right), T\left(r, f_{2}\right)\right\}$. If $\alpha_{1} f_{1}+\alpha_{2} f_{2}=1$, then

$$
T\left(r, f_{i}\right)<\bar{N}\left(r, \frac{1}{f_{1}}\right)+\bar{N}\left(r, \frac{1}{f_{2}}\right)+\bar{N}\left(r, f_{\imath}\right)+o\{T(r)\} . \quad(r \notin E ; i=1,2)
$$

LEMMA 3 ([5]). Let $f,(j=1,2,3)$ be three nonconstant meromorphic functions, satısfyıng $\sum_{j=1}^{3} f_{j} \equiv 1$. And let $g_{1}=-f_{1} / f_{2}, g_{2}=1 / f_{2}, g_{3}=-f_{3} / f_{2}$. If $f_{3}$ $(j=1,2,3)$ are linearly independent, then $g_{j}(j=1,2,3)$ also are linearly independent.

\section{Proof of Theorems and Corollary.}

The proof of theorem 1 . In fact, let

$$
\frac{f-\mu}{g-\lambda}=h
$$

and $T(r)=\max \{T(r, f), T(r, g)\}$. Then the poles and zeros of $h$ only occur at the poles of $\mu$ and $\lambda$ at most by $E(\infty, f)=E(\infty, g)$ and $E(\mu, f)=E(\lambda, g)$. Hence

$$
N(r, h)+N\left(r, \frac{1}{h}\right)=o\{T(r)\} .
$$


Next, from (1) we get

$$
f-\mu=g h-\lambda h .
$$

We complete the proof by the following two cases:

CASE 1. $h \equiv k$ (const.), then when $k \neq \equiv / \lambda$ we have from (3)

$$
\frac{f}{\mu-\lambda k}-\frac{k}{\mu-\lambda k} g=1 \text {. }
$$

From Lemma 2 (by taking $\left.\alpha_{1}=(1 / \mu-\lambda k) \not \equiv 0, \alpha_{2}=-(k / \mu-\lambda k) \not \equiv 0\right)$ we get

$$
T(r, f)<N\left(r, \frac{1}{f}\right)+N\left(r, \frac{1}{g}\right)+\bar{N}(r, f)+o\{T(r)\}, \quad(r \notin E) .
$$

On the other hand, from (4) we know that

$$
T(r, g) \leqq(1+o(1)) T(r, f)
$$

so that

$$
o\{T(r)\}=o\{T(r, f)\}
$$

Hence

$$
\begin{aligned}
T(r, f)< & {[2-\delta(0, f)-\Theta(\infty, f)] T(r, f) } \\
& +[1-\delta(0, g)] T(r, g)+o\{T(r)\} \quad(r \notin E) \\
\leqq & {[3-\delta(0, f)-\Theta(\infty, f)-\delta(0, g)] T(r, f)+o\{T(r)\}, \quad(r \notin E) . }
\end{aligned}
$$

Since

$$
3-\delta(0, f)-\Theta(\infty, f)-\delta(0, g)<\frac{3}{2}-\delta(0, g)<1,
$$

by (5) and (6) we deduce that

$$
T(r, f)=o\{T(r, f)\}, \quad(r \notin E)
$$

which is a contradiction.

It shows that if $h$ is a constant function, $h$ must be equal to $\mu / \lambda$. Hence we obtain from (1)

$$
\frac{f}{\mu}=\frac{g}{\lambda} .
$$

CASE 2. $h \not \equiv$ constant, let

then from (3)

$$
f_{1}=\frac{f}{\mu}, \quad f_{2}=\frac{\lambda}{\mu} h, \quad f_{3}=-\frac{g}{\mu} h .
$$

$$
\sum_{j=1}^{3} f_{j} \equiv 1
$$


Suppose $f_{0}(j=1,2,3)$ are linearly independent, it is easy to see from Lemma 1 that

$$
\begin{aligned}
T(r, f)< & N\left(r, \frac{1}{f}\right)+N\left(r, \frac{1}{g}\right)+N(r, f)+N(r, D) \\
& -\sum_{j=1}^{3} N\left(r, f_{j}\right)+o\{T(r)\}, \quad(r \notin E)
\end{aligned}
$$

where

From (7) we get

$$
D=\left|\begin{array}{lll}
f_{1} & f_{2} & f_{3} \\
f_{1}^{\prime} & f_{2}^{\prime} & f_{3}^{\prime} \\
f_{1}^{\prime \prime} & f_{2}^{\prime \prime} & f_{3}^{\prime \prime}
\end{array}\right|
$$

Hence

$$
D=\left|\begin{array}{lll}
f_{1} & f_{2} & 1 \\
f_{1}^{\prime} & f_{2}^{\prime} & 0 \\
f_{1}^{\prime \prime} & f_{2}^{\prime \prime} & 0
\end{array}\right|=\left|\begin{array}{cc}
f_{1}^{\prime} & f_{2}^{\prime} \\
f_{1}^{\prime \prime} & f_{2}^{\prime \prime}
\end{array}\right| .
$$

Thus

$$
N(r, D) \leqq N(r, f)+2 \bar{N}(r, f)+o\{T(r)\}
$$

$$
N(r, f)+N(r, D)-\sum_{j=1}^{3} N\left(r, f_{j}\right) \leqq 2 \bar{N}(r, f)+o\{T(r)\} .
$$

Then, from (8) we obtain

$$
T(r, f)<N\left(r, \frac{1}{f}\right)+N\left(r, \frac{1}{g}\right)+2 \bar{N}(r, f)+o\{T(r)\} . \quad(r \notin E)
$$

Next, according to Lemma 3 we know that $g_{1}=-f / \lambda h, g_{2}=\mu / \lambda h, g_{3}=g / \lambda$ are also linearly independent. Similary, we can get

$$
T(r, g)<N\left(r, \frac{1}{f}\right)+N\left(r, \frac{1}{g}\right)+2 \bar{N}(r, g)+o\{T(r)\}, \quad(r \notin E) .
$$

By (10) and (11) we have

$$
\begin{aligned}
T(r, f)+T(r, g)< & 2\left[N\left(r, \frac{1}{f}\right)+\bar{N}(r, f)\right]+2\left[N\left(r, \frac{1}{g}\right)+\bar{N}(r, g)\right] \\
& +o\{T(r)\} \quad(r \notin E) \\
\leqq & 2[2-\delta(0, f)-\Theta(\infty, f)] T(r, f)+o\{T(r)\} \\
& +2[2-\delta(0, g)-\Theta(\infty, g)] T(r, g), \quad(r \notin E)
\end{aligned}
$$

but

$$
2[2-\delta(0, f)-\Theta(\infty, f)]<1
$$


and

$$
2[2-\delta(0, g)-\Theta(\infty, g)]<1 .
$$

Hence from (12) we deduce that

$$
T(r)=o\{T(r)\}, \quad(r \notin E) .
$$

This is a contradiction.

It shows that $f_{\jmath}(\jmath=1,2,3)$ are linearly dependent, i. e., there exist three constants $\left(c_{1}, c_{2}, c_{3}\right) \neq(0,0,0)$ such that

$$
c_{1} f_{1}+c_{2} f_{2}+c_{3} f_{3}=0 \text {. }
$$

If $c_{1}=0$, since $h \not \equiv$ constant and $h \not \equiv$ constant, from (13) we get

$$
g=\frac{c_{2}}{c_{3}} \lambda,
$$

contradicting given condition $T(r, \lambda)=o\{T(r, g)\}$. Hence $c_{1} \neq 0$. Then, combining (7) and (13) we have

$$
\left(1-\frac{c_{2}}{c_{1}}\right) \frac{\lambda}{\mu} h+\left(\frac{c_{3}}{c_{1}}-1\right) \frac{g}{\mu} h=1,
$$

We assert that $1-\left(c_{2} / c_{1}\right)=0$. Otherwise, then $1-\left(c_{2} / c_{1}\right) \neq 0$. If $\left(c_{3} / c_{1}\right)-1 \neq 0$, we get by Lemma 2

$$
\begin{aligned}
T(r, g) & <N\left(r, \frac{1}{g}\right)+\bar{N}(r, g)+o\{T(r, g)\} \quad(r \notin E) \\
& \leqq[2-\delta(0, g)-\Theta(\infty, g)] T(r, g)+o\{T(r, g)\} \quad(r \notin E) \\
& \leqq \frac{1}{2} T(r, g)+o\{T(r, g)\}, \quad(r \notin E) .
\end{aligned}
$$

It is impossible. If $\left(c_{3} / c_{1}\right)-1=0$, then

$$
h=\frac{c_{1}}{c_{1}-c_{2}} \cdot \frac{\mu}{\lambda}
$$

from (1) we get

$$
\frac{f}{\mu w}-\frac{g}{\lambda w} \cdot \frac{c_{1}}{c_{1}-c_{2}}=1
$$

where $w=1-\left(c_{1} / c_{1}-c_{2}\right)$. Here we may assume $w \neq 0$, because, if $w=0$, then we have $f / \mu \equiv g / \lambda$. By Lemma 2 we have

$$
\begin{aligned}
T(r, f) & <N\left(r, \frac{1}{f}\right)+N\left(r, \frac{1}{g}\right)+\bar{N}(r, f)+o\{T(r, f)\} \quad(r \notin E) \\
& \leqq[3-\delta(0, f)-\delta(0, g)-\Theta(\infty, f)] T(r, f)+o\{T(r, f)\}, \quad(r \notin E)
\end{aligned}
$$

but 


$$
3-\delta(0, f)-\delta(0, g)-\Theta(\infty, f)<1 \text {. }
$$

It is also impossible. Thus $1-\left(c_{2} / c_{1}\right)=0$. i. e.,

$$
\frac{g}{\mu} h=\frac{c_{1}}{c_{3}-c_{1}} \text {. }
$$

Substituting this into (1) we obtain

$$
\frac{f}{\mu}=\frac{c_{3}}{c_{3}-c_{1}}-\frac{\lambda}{\mu} h \text {. }
$$

It is easy to see that $c_{3}=0$ by Lemma 2 . Hence from (15) and (16) we get, respectively ;

$$
f=-\lambda h, \quad g=-\frac{\mu}{h}
$$

i. e.,

$$
f \cdot g=\mu \cdot \lambda .
$$

This complete the proof of Theorem 1 .

The proof of Theorem 2. First, by Theorem 1 we get

$$
\frac{f}{\varphi_{1}}=\frac{g}{h_{1}}
$$

or

and

$$
f \cdot g=\varphi_{1} \cdot h_{1},
$$

$$
\frac{f}{\varphi_{2}}=\frac{g}{h_{2}}
$$

or

$$
f \cdot g=\varphi_{2} \cdot h_{2},
$$

from (17) and (19) we have

$$
\frac{f}{\varphi_{1}}=\frac{g}{h_{1}} \quad \text { and } \quad \frac{\varphi_{1}}{h_{1}}=\frac{\varphi_{2}}{h_{2}},
$$

from (18) and (20) we have

$$
f \cdot g=\varphi_{1} \cdot h_{1} \quad \text { and } \quad \varphi_{1} \cdot h_{1}=\varphi_{2} \cdot h_{2} .
$$

On the other hand, from (17) and (20) or (18) and (19) we obtain, respectively;

$$
T(r, f)=o\{T(r, f)\}, \quad T(r, g)=o\{T(r, g)\},
$$

which is a contradiction. This completes the proof of Theorem 2 .

The proof of Corollary. Let 


$$
f=\mu_{1} e^{\alpha}+\mu_{2}, \quad g=\lambda_{1} e^{\beta}+\lambda_{2},
$$

where $\alpha$ and $\beta$ are two entire functions, $\mu_{2}$ and $\lambda_{2}(i=1,2)$ are meromorphic functions, satisfying $\mu_{1} \neq 0, \mu_{2} \neq$ const., $\lambda_{1} \neq 0$ and $\lambda_{2} \neq$ const.,

$$
T\left(r, \mu_{\imath}\right)=o\left\{T\left(r, e^{\alpha}\right)\right\}, \quad T\left(r, \lambda_{\imath}\right)=o\left\{T\left(r, e^{\beta}\right)\right\} . \quad(i=1,2)
$$

Again let

$$
f^{*}=\frac{f-\mu_{2}}{\mu_{1}}, \quad g^{*}=\frac{g-\lambda_{2}}{\lambda_{1}},
$$

then

Obviouly,

$$
f^{*}=e^{\alpha}, \quad g^{*}=e^{\beta} .
$$

and

$$
E\left(\infty, f^{*}\right)=E\left(\infty, g^{*}\right)
$$

$$
\begin{aligned}
& \delta\left(0, f^{*}\right)+\Theta\left(\infty, f^{*}\right)=2>\frac{3}{2}, \\
& \delta\left(0, g^{*}\right)+\Theta\left(\infty, g^{*}\right)=2>\frac{3}{2} .
\end{aligned}
$$

From $E\left(c_{\imath}, f\right)=E\left(c_{\imath}, g\right)(i=1,2)$ we have

$$
E\left(c_{i}-\mu_{2}, \mu_{1} f^{*}\right)=E\left(c_{i}-\lambda_{2}, \lambda_{1} g^{*}\right) . \quad(i=1,2) .
$$

By Theorem 2 we have

$$
\frac{\mu_{1} f^{*}}{c_{1}-\mu_{2}}=\frac{\lambda_{1} g^{*}}{c_{1}-\lambda_{2}}
$$

and

$$
c_{1}-\mu_{2} / c_{1}-\lambda_{2}=c_{2}-\mu_{2} / c_{2}-\lambda_{2},
$$

or

$$
\mu_{1} f^{*} \lambda_{1} g^{*}=\left(c_{1}-\mu_{2}\right) \cdot\left(c_{1}-\lambda_{2}\right)
$$

and

$$
\left(c_{1}-\mu_{2}\right) \cdot\left(c_{1}-\lambda_{2}\right)=\left(c_{2}-\mu_{2}\right) \cdot\left(c_{2}-\lambda_{2}\right) .
$$

If (i) holds, then from (21) and (22) we obtain

$$
\mu_{2}=\lambda_{2}
$$

and

$$
\frac{f-\mu_{2}}{\mu_{1}}=\frac{g-\lambda_{2}}{\lambda_{1}} \cdot \frac{\lambda_{1}}{\mu_{1}},
$$

i. e., $f \equiv g$.

If (ii) holds, then from (23) and (24) we obtain 


$$
\left(\mu_{2}+\lambda_{2}\right)=\left(c_{1}+c_{2}\right)
$$

and

$$
\left(f-\mu_{2}\right) \cdot\left(g-\lambda_{2}\right)=\left(c_{1}-\mu_{2}\right) \cdot\left(c_{1}-\lambda_{2}\right) .
$$

Hence

$$
\mu_{1} e^{\alpha} \cdot \lambda_{1} e^{\beta}=\left(\lambda_{2}-c_{2}\right)\left(c_{1}-\lambda_{2}\right)
$$

Thus

$$
f=\frac{\left(c_{2}-\lambda_{2}\right)\left(c_{1}-\lambda_{2}\right)}{\lambda_{1} e^{\beta}}+\left(c_{1}+c_{2}-\lambda_{2}\right) .
$$

Obviously, only letting

$$
\lambda_{1}=\frac{h}{1-\lambda}, \quad e^{\beta}=e^{\phi}, \quad \lambda_{2}=\frac{c_{1}-c_{2} \lambda}{1-\lambda},
$$

we can deduce the conjecture of C. C. Yang.

I thank a lot for the useful suggestion of referees.

\section{REFERENCES}

[1] C.C. YANG, On Meromorphic Functions Taking the Same Values at the Same Points, Kodai Math. Sem. Rep., 28 (1977), 300-309.

[2] F. Gross, Factorization of meromorphic functions, U.S. Govt. Math. Res. Center, 1972.

[3] W.K. Hayman, Meromorphic functions, Oxford, 1964.

[4] YI HongXun, Meromorphic functions with two deficient values, Acta Math. Sinica., (Chinese) $30: 5$ (1987), 588-597.

[5] YI HongXun, Meromorphic functions that share two or three values, Kodai Math. J., 13 (1990), 363-372.

Department of Mathematics,

Ningde Teachers College,

Ningde, Fujian, P.R. China 